\begin{tabular}{|c|c|c|c|c|c|c|c|}
\hline Persistence & $5(31 \%)$ & $2(18 \%)$ & $2(40 \%)$ & $7(64 \%)$ & $3(75 \%)$ & $0(0 \%)$ & \\
\hline Surgery & $4(25 \%)$ & $3(27 \%)$ & $2(40 \%)$ & $327 \%)$ & $0(0 \%)$ & $0(0 \%)$ & \\
\hline \multicolumn{8}{|c|}{ Number of previous biologics } \\
\hline 0 & $15(25.4 \%)$ & $5(8.5 \%)$ & $1(1.7 \%)$ & $5(8.5 \%)$ & $3(5.1 \%)$ & $0(0 \%)$ & $6(10.2 \%)$ \\
\hline 1 & $0(0 \%)$ & $6(10.2 \%)$ & $2(3.4 \%)$ & $3(5.1 \%)$ & $1(1.7 \%)$ & $0(0 \%)$ & $6(10.2 \%)$ \\
\hline 2 & $1(1.7 \%)$ & $0(0 \%)$ & $2(3.4 \%)$ & $3(5.1 \%)$ & $0(0 \%)$ & $1(1.7 \%)$ & $4(6.8 \%)$ \\
\hline$\chi^{2} p$-value & $<0.0001$ & 0.042 & 0.424 & 0.954 & 0.422 & 0.195 & 0.592 \\
\hline \multicolumn{8}{|c|}{ Disease Location } \\
\hline E1 & $2(3.4 \%)$ & $0(0 \%)$ & $0(0 \%)$ & $2(3.4 \%)$ & $1(1.7 \%)$ & $1(1.7 \%)$ & $1(1.7 \%)$ \\
\hline E2 & $9(15.3 \%)$ & $6(10.2 \%)$ & $1(1.7 \%)$ & $5(8.5 \%)$ & $3(5.1 \%)$ & $0(0 \%)$ & 7 (11.9\%) \\
\hline E3 & $5(8.5 \%)$ & $5(8.5 \%)$ & $4(6.8 \%)$ & $4(6.8 \%)$ & $0(0 \%)$ & $0(0 \%)$ & $8(13.6 \%)$ \\
\hline$\chi^{2}$ p-value & 0.282 & 0.324 & 0.295 & 0.644 & 0.142 & 0.023 & 0.717 \\
\hline
\end{tabular}

(median, interquartile ranges (IQR), Mann-Whitney U- test); categorical variables (frequency, percentages, chi-square test).

Results Data from 90 CD and 103 UC patients was analysed. Prior biologic exposure (Adalimumab 57.5\% (111/193); Infliximab 46.6\% (90/193); Ustekinumab in CD 42.2\% (38/90)) was high overall.

Total all-cause VDZ discontinuation rate was $74.4 \%(67 / 90)$ in $\mathrm{CD}$ and $56.3 \%(58 / 103)$ in UC with the majority discontinuing within year 1 (CD: $n=44$; UC: $n=35$ ) as compared to years 2,3 or greater (CD: $n=13,8,2$; UC: $n=11,6$, 7). Of these patients, $77.6 \%(52 / 67)$ with $\mathrm{CD}$ and $63.8 \%(37 /$ 58 ) with UC required another biologic. A majority with $\mathrm{CD}$ persisted on subsequent Ustekinumab $(68 \%$; 30/44) or antiTNF $(71 \% ; 5 / 7)$ by the end of the study period. In UC, $26 \%$ (7/27) persisted on an anti-TNF and 64\% (7/11) on Tofacitinib (Table 1).

17 patients were retreated with VDZ having discontinued it for remission (CD: $n=7$; UC: $n=10$ ). The median duration of VDZ retreatment was 164 [IQR: 87-189] weeks for $\mathrm{CD}$ and 165 [IQR: 126-205] weeks for UC (duration followup from first VDZ discontinuation for CD: 46 [IQR: 30-56] weeks; UC: 46 [IQR: 16-77] weeks). Of this larger cohort, there was no significant difference in relapse rate on VDZ retreatment between CD and UC $(14 \%(n=1)$ vs. $30 \%(n$ $=3), p=0.6029)$ ).

A large minority of patients underwent subsequent surgery (CD: $38.8 \%$ (26/67); UC: $29.3 \%$ (17/59) with the median interval to surgery greatest in UC (CD: 21 (IQR: 9-71) vs. UC 63 (IQR: 12-84) weeks, $p=0.2409$ ). Most CD patients who had surgery post VDZ $(61.5 \%, 16 / 26)$ had had 2 prior biologics as compared to none who had VDZ first line $(p=$ 0.184 ) whereas in UC, biologic exposure had less impact on surgery rate $(p=0.592)$.

Conclusions Our data suggests VDZ recapture for relapse following discontinuation of therapy for remission, sequencing Ustekinumab after VDZ in complex/refractory CD and antiTNF therapy before or after VDZ in UC are options in the management algorithm of IBD.

\section{PMO-13 ESTABLISHING TRENDS IN LUMINAL INFLAMMATION TOWARDS THE END OF LIFE IN PATIENTS WITH LONGSTANDING IBD}

Alexander Robertson*, Gareth-Rhys Jones. Edinburgh IBD Unit, Western General Hospital, Edinburgh, UK

10.1136/gutjnl-2021-BSG. 152
Introduction Inflammatory bowel disease (IBD) is a chronic condition characterised by unpredictable mucosal inflammation which can severely impact a patient's quality of life. Due to compounding prevalence and an aging population, more people are dying with IBD. This study aims to describe IBD activity in a prevalent cohort in the years before death using faecal calprotectin (FC).

Method The NHS Lothian IBD registry is a rigorously validated, prevalent IBD cohort that has been shown to be circa 95\% complete for the approximate one million population of the Lothians, Scotland. Data on $>11 \mathrm{k}$ patients on date of diagnosis, death, IBD subtype, and serial FC measurements are held since 2003.

Patients with disease duration $\geq 8$ years who had died and had $\geq 1$ FC in the 5 years preceding death were identified. FC was correlated to patient demographics, IBD subtype, time before death and cause of death.

Results 261 deceased individuals (136 ulcerative colitis (UC) and 125 with Crohn's disease (CD)) were identified. 28 were excluded, having died of small bowel or colonic disease (16 with UC and 12 with CD).

Mean age at death was 74.47 (SD 13.6) years in UC cohort (49.2\% female) versus 70.47 (SD 14.3) in CD cohort (57.5\% female).

The median FC decreased in those with UC (from 705 to $438 \mathrm{ug} / \mathrm{g}$ ) but rose in those with CD (210 to $310 \mathrm{ug} / \mathrm{g})$ in the 5 years prior to death, (Figure 1).

Deaths from acute illness/accident, chronic disease (or acute on chronic illness) and malignancy were compared. FC measurements remained constant for those dying from both acute illness and chronic disease, although in both UC and CD an

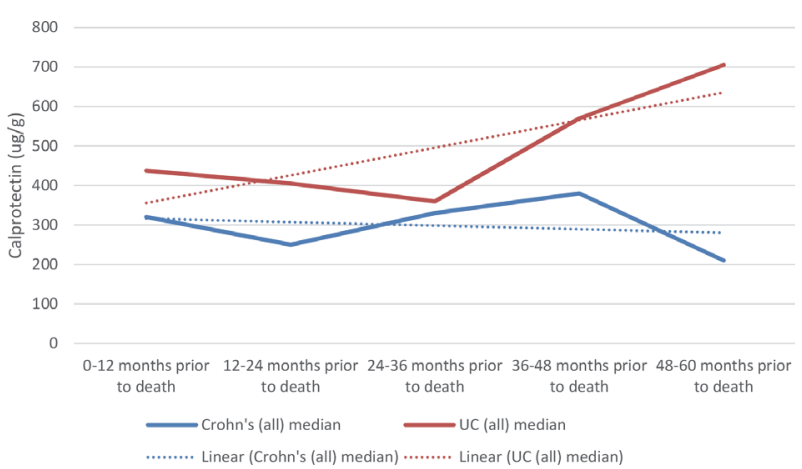

Abstract PM0-13 Figure 1 Median FC levels in the years prior to death 
improvement in median FC levels was seen in those dying from malignancy (1130 to $250 \mathrm{ug} / \mathrm{g}$ in UC and 588 to $490 \mathrm{ug} /$ $\mathrm{g}$ in $\mathrm{CD})$.

When stratified by age of death the FC measurements were similar between age groups.

Conclusions Serious and fatal non-IBD related disease does not seem to lead to spontaneous improvement or deterioration in luminal inflammation prior to death, with no significant 'burn out' of IBD activity seen. For patients approaching the end of their life the aim of treatment focuses on maximising quality of life, but there remains variation in practice around stopping or continuing IBD therapies, and further research in this area is required. This study suggests that for patients with IBD an individualised approach is required, and for many, good palliative care will include active and aggressive management of their IBD.

\section{PMO-14 USE OF NON-INVASIVE SURROGATE MARKERS TO MONITOR VEDOLIZUMAB THERAPY IN INFLAMMATORY BOWEL DISEASE}

${ }^{1}$ John Barragry*, 'Konstantinos Fragkos, 'Greg Sebepos-Rogers, 'Hajeena Saravanapavan, ${ }^{2}$ Hui Fen Koo, ${ }^{2}$ Ho Juen Ko, ${ }^{1}$ loanna Parisi, ${ }^{1}$ Edward Seward, ${ }^{1}$ Sara McCartney, ${ }^{1}$ Stuart Bloom, 'Shameer Mehta, 'Farooq Rahman. 'Dept. Gastroenterology, University College Hospital, London, UK; ${ }^{2}$ University College London Medical School, London, UK

\subsection{6/gutjnl-2021-BSG.153}

Introduction We provide long-term data on the effectiveness of Vedolizumab (VDZ) in the management of inflammatory bowel disease (IBD) by reviewing the response of surrogate markers of disease activity to VDZ therapy, including HarveyBradshaw Index (HBI) for Crohn's disease (CD) and Simple Colitis Clinical Activity Index (SCCAI) for ulcerative colitis (UC), c-reactive protein (CRP), and faecal calprotectin (FCP).

Methods A consecutive cohort of 193 adult IBD patients (90 $\mathrm{CD}$ and 103 UC/IBD-unspecified) who commenced VDZ between May 2015 and June 2019 at a tertiary IBD centre was retrospectively reviewed. Markers of disease at baseline, 1 year $(\mathrm{n}=193), 2$ years $(\mathrm{n}=157)$ and 3 years $(\mathrm{n}=104)$ were assessed. Statistical analyses were performed using SPSS 25.0 , with significance assigned to a p-value of $<0.05$ in bootstrap t-testing.

Results Overall, $77.8 \%$ and $93.2 \%$ of patients with $\mathrm{CD}$ and UC respectively had undergone more than one baseline assessment modality prior to VDZ therapy. At baseline for CD and UC patients respectively; a) median disease activity index (DAI) score was HBI 5 (interquartile range (IQR): 2-8) and SCCAI 5 (IQR: 3-8), b) median CRP was $9.9 \mathrm{mg} / \mathrm{L}$ (IQR: 3.73-22.5 $\mathrm{mg} / \mathrm{L}$ ) and $4.8 \mathrm{mg} / \mathrm{L}$ (IQR: $1.5-10.5 \mathrm{mg} / \mathrm{L}$ ), c) median FCP was $656.5 \mu \mathrm{g} / \mathrm{g}$ (IQR: 301-1472 $\mu \mathrm{g} / \mathrm{g}$ ) and 1190 $\mu \mathrm{g} / \mathrm{g}$ (IQR: $386.5-2639 \mu \mathrm{g} / \mathrm{g}$ ).

Following initiation of VDZ therapy, DAI scores indicated a favourable response in patients with colonic CD disease and all UC disease subtypes from visit 2 onwards compared to baseline $(p<0.05$, Figure $1 A, B)$. There was a significant fall in CRP in colonic but not ileal CD patients $(p<0.05$ at multiple timepoints after visit 4 in Year 1), a trend that persisted during year 1 and extended up to year 3 (Figure 1C). In UC patients, a significant fall was present from visit 3 in Year 1 and persisted in E1 and E2 UC patients compared to baseline $(p<0.05$, Figure 1D). There was a significant reduction in FCP over time in patients with colonic CD and E3 UC $(\mathrm{P}<0.01$, Figure 1E,F).

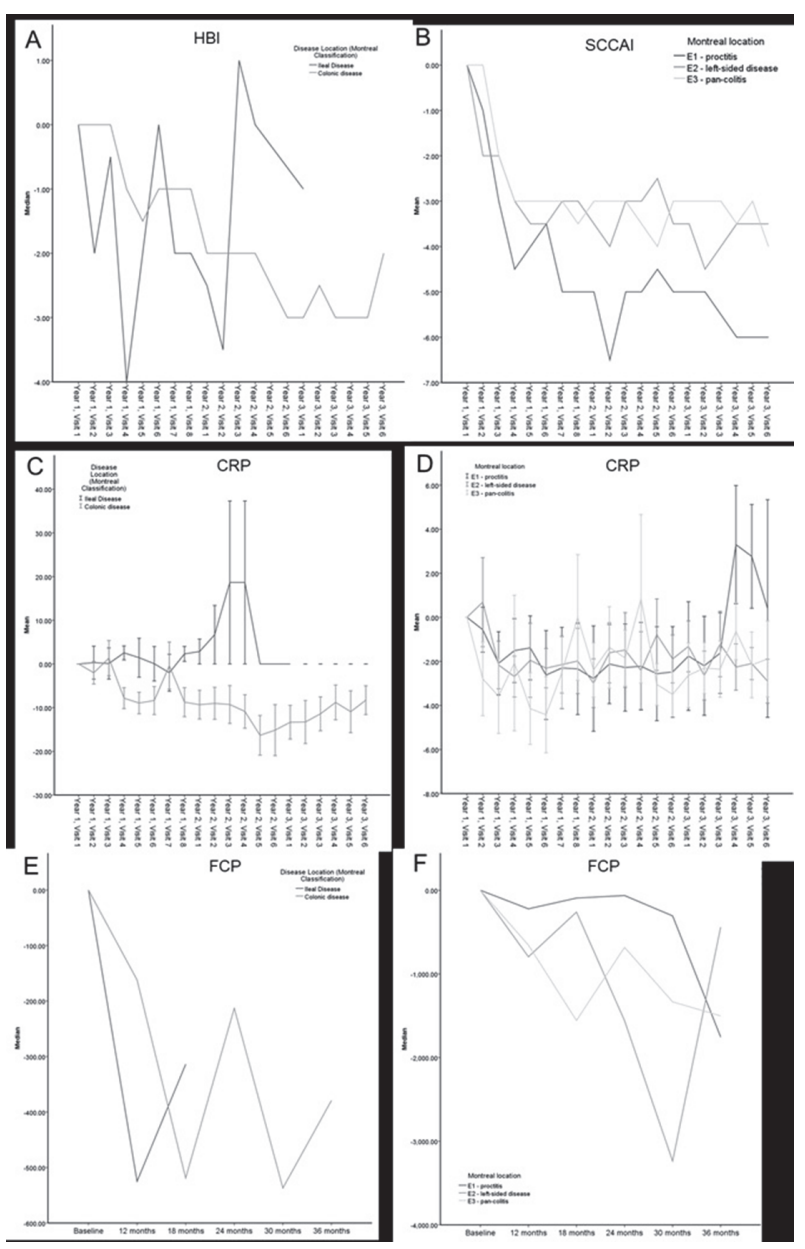

Abstract PM0-14 Figure 1

Conclusion In our single centre retrospective cohort analysis of 193 adult IBD patients, we observed a clinically and statistically significant improvement in markers of disease activity, including DAI scores, CRP and FCP. These surrogate markers allow non-invasive monitoring of VDZ response, particularly in patients with colonic CD and UC.

\section{PMO-15 TRANSITIONING FROM INTRAVENOUS TO SUBCUTANEOUS VEDOLIZUMAB IN PATIENTS WITH INFLAMMATORY BOWEL DISEASE (TRAVELESS)}

${ }^{1}$ Esther Ventress*, ${ }^{*}$ Sohail Rahmany, ${ }^{1}$ David Young, 'Marion Bettey, ${ }^{1}$ Clare Harris, ${ }^{2}$ Helen Moyses, ${ }^{1}$ Trevor Smith, ${ }^{1}$ Richard Felwick, ${ }^{1}$ Markus Gwiggner, ${ }^{1,3}$ Fraser Cummings. 'University Hospital Southampton NHS Foundation Trust, Southampton, UK; ${ }^{2}$ NIHR Biomedical Research Unit, Southampton General Hospital, Southampton, UK; ${ }^{3}$ Faculty of Medicine, University of Southampton, Southampton, UK

\subsection{6/gutjnl-2021-BSG.154}

Introduction In May 2020, subcutaneous (SC) vedolizumab was approved for use in Inflammatory Bowel Disease (IBD). Patients with IBD have a number of risk factors for a poor outcome from SARS-CoV-2 infection and managing this risk by reducing hospital visits is crucial. Currently there is no information on the process or outcomes of transitioning patients established on intravenous (IV) vedolizumab to SC.

Methods This is a prospective service evaluation of adult patients who are either stable on IV vedolizumab or have 